\title{
Uptake of more important mineral components by common field weeds on loess soils
}

\author{
LESZEK MALICKI and CZESEAWA BERBECIOWA
}

Department of Bases of Agrotechnics, Agricultural Academy of Lublin, Akademicka 13, 20-934 Lublin, Poland

(Received: October 10, 1984)

\begin{abstract}
We have determined the contents of $\mathrm{N}, \mathrm{P}, \mathrm{K}, \mathrm{Ca}, \mathrm{Mg}, \mathrm{Na}, \mathrm{Fe}, \mathrm{Cu}, \mathrm{Mn}$ and $\mathrm{Zn}$ in winter wheat, spring barley, sugar beets and winter rape, as well as in the most common weed species infesting these crops. It was established that the percentage of mineral components in the dry matter of the majority of weeds is higher than in that of the cultivated plants. The most dangerous weed species competing with plants for the investigated nutrients were: Chenopodium album, Cirsium arvense, Convolvulus arvensis, Polygonum convolvulus, Sonchus arvensis and Stellaria media.
\end{abstract}

\section{INTRODUCTION}

One of the most important forms of damage caused by weeds consists in the fact that they rob plants of mineral nutrients. The scope of the phenomenon can be deduced from some remarks scattered throughout publications mentioning the "greed" of particular weed species (Li-Sun-Żun, 1962; Mikłosz-Wiśniewska and Olkowski, 1981; Mowszowicz, 1975 after Korsmo), or of weeds as a group (Oświt and Sapek, 1982; Shukla and Vimal, 1969). In the available literature the problem is discussed more thoroughly by Ś więtochowski and Sońta (1962) and by Czuba and Wróbel (1983), still they do not exhaust the issue. This fact constituted the incentive to the research presented in our paper.

\section{MATERIAL AND METHODS}

The research material consisted of the above-ground parts and roots of cultivated plants, as well as of weeds infesting the crops of winter wheat, spring barley, sugar beets and winter rape grown in the production fields of the Agricultural Experimental Station in Czesławice in the Lublin district.

The soils within the station area developed from deep loess into brown, graybrown podsolic and podsolic, slightly eroded and deluvial soils. They are charac- 
terized as slightly acid soils with high contents of available phosphorus and magnesium, but deficient in potassium. The depth of the humus layer does not exceed $30 \mathrm{~cm}$. Thus, they may be considered representative for that type of soil conditions. Similarly, the average precipitation and air temperature patterns in the research period (1974-1976) did not differ too much from long term figures.

The analyzed cultivated plants were harvested when ripe, while weeds - at the time of seed initiation. Each species was represented by a sample containing from several dozen up to 200 individuals, the count being inversely proportional to the mass of an individual plant.

The nitrogen content in the plant material was determined by means of Kjeldahl's method, in samples wet-mineralized, whereas the contents of other components, i.e. $\mathrm{P}, \mathrm{K}, \mathrm{Ca}, \mathrm{Mg}, \mathrm{Na}, \mathrm{Fe}, \mathrm{Cu}, \mathrm{Mn}$ and $\mathrm{Zn} \mathrm{-} \mathrm{in} \mathrm{dry-mineralized} \mathrm{samples.}$ The phosphorus content was determined colorimetrically, while the other elements - using an atomic absorption spectrometer.

Additionally, the air dry, and then absolutely dry mass of plants was measured in order to calculate the uptake of particular elements per individual representing the investigated species.

\section{RESULTS}

Particular weed species infesting winter wheat represented various chemical compositions. Still, the majority was characterized by a significantly greater content of mineral elements as compared with wheat (Table 1). The difference was especially marked in the case of Chenopodium album, Sonchus arvensis, Polygonum convolvulus, Polygonum lapathifolium and Convolvulus arvensis, with respect to nitrogen in particular (Table 1). It must be noted, however, that the uptake of nutrients per individual plant species presented a slightly different picture (Table 2). Only a few species turned out to possess a richer nutrient uptake than wheat; the contents of the majority of components, as well as their total quantities in Centaurea cyanus, Cirsium arvense and Convolvulus arvensis exceeded those in wheat (not in every case significantly in statistical terms).

The chemical composition of the weeds infesting spring barley did not vary as much as in the case of wheat-infesting weeds. Nevertheless, the concentration of the major mineral components in the dry matter of Polygonum convolvulus, Chenopodium album, Convolvulus arvensis, Sonchus arvensis, Sonchus asper and Stellaria media was higher than in the other barley-infesting weeds and also than in barley itself (Table 3). Only Vicia hirsuta and Vicia angustifolia were quite unsurpassed in their nitrogen content, which was to be expected.

The highest total uptake of the investigated components was in Sonchus arvensis (Table 4). Considerable quantities of these macroelements were found in Convolvulus arvensis, Galeopsis tetrahit, Sonchus asper, Vicia hirsuta and Vicia angustifolia. However, as compared with barley, none of the weeds contained as much of the 
Table 1

Contents of some mineral components in winter wheat (grain + straw + roots) and its weeds (the average of 1974-1976)

\begin{tabular}{|c|c|c|c|c|c|c|c|c|c|c|}
\hline \multirow{2}{*}{ Species } & \multicolumn{5}{|c|}{ Percent of dry matter } & \multicolumn{5}{|c|}{$\mathrm{mg} / \mathrm{kg}$} \\
\hline & $\mathrm{N}$ & $\mathbf{P}$ & $\mathbf{K}$ & $\mathrm{Ca}$ & $\mathrm{Mg}$ & $\mathrm{Na}$ & $\mathrm{Fe}$ & $\mathrm{Cu}$ & $\mathrm{Mn}$ & $\mathrm{Zn}$ \\
\hline Triticum aestivum $\mathrm{L}$. & 1.22 & 0.27 & 0.80 & 1.06 & 0.12 & 0.03 & 197.3 & 4.2 & 23.0 & 22.0 \\
\hline Capsella bursa-pastoris (L.) Med. & 1.63 & 0.25 & 2.00 & 1.08 & 0.17 & 0.02 & 45.5 & 7.4 & 33.5 & 28.0 \\
\hline Centaurea cyanus $\mathrm{L}$. & 1.25 & 0.29 & 1.44 & 1.06 & 0.15 & 0.04 & 112.3 & 11.6 & 49.6 & 31.3 \\
\hline Chenopodium album $\mathrm{L}$. & 2.63 & 0.48 & 3.69 & 2.08 & 0.45 & 0.11 & 400.6 & 6.7 & 91.6 & 45.7 \\
\hline Galeopsis tetrahit $\mathrm{L}$. & 1.45 & 0.41 & 1.92 & 1.26 & 0.19 & 0.06 & 656.3 & 5.7 & 86.6 & 65.8 \\
\hline Myosotis arvensis (L.) Hill. & 1.58 & 0.59 & 2.83 & 1.62 & 0.24 & 0.11 & 653.3 & 12.6 & 68.0 & 35.0 \\
\hline Polygonum convolvulus $\mathbf{L}$. & 2.53 & 0.37 & 2.03 & 1.60 & 0.53 & 0.04 & 266.6 & 9.2 & 66.3 & 31.8 \\
\hline Polygonum lapathifolium $\mathrm{L}$. & 2.18 & 0.51 & 2.08 & 0.94 & 0.36 & 0.15 & 558.6 & 15.4 & 111.3 & 60.7 \\
\hline Sonchus arvensis L. & 2.35 & 0.54 & 3.58 & 2.22 & 0.58 & 0.18 & 377.6 & 11.0 & 87.6 & 89.1 \\
\hline Siellaria media Vill. & 1.78 & 0.53 & 3,90 & 1.06 & 0.28 & 0.23 & 1154.3 & 6.0 & 145.3 & 30.1 \\
\hline $\mathrm{LSD}_{0.05}$ & 1.10 & 0.26 & 1.69 & 1.28 & 0.21 & 0.15 & 843.2 & 11.7 & 77.4 & 39.6 \\
\hline
\end{tabular}


Table 2

Uptake of mineral components by winter wheat and its weeds, in mg per plant (the average of 1974-1976)

\begin{tabular}{|c|c|c|c|c|c|c|c|c|c|c|c|}
\hline Species & $\mathbf{N}$ & $\mathbf{P}$ & $\mathrm{K}$ & $\mathrm{Ca}$ & $\mathrm{Mg}$ & $\mathrm{Na}$ & $\mathrm{Fe}$ & $\mathrm{Cu}$ & $\mathrm{Mn}$ & $\mathrm{Zn}$ & Sum \\
\hline Triticum aestivum $\mathrm{L}$. & 99.84 & 22.38 & 63.80 & 58.64 & 9.96 & 2.72 & 1.70 & 0.05 & 0.19 & 0.09 & 259.37 \\
\hline Agropyron repens (L.) P.B. & 25.72 & 3.91 & 18.15 & 19.67 & 2.22 & 0.56 & 0.22 & 0.01 & 0.05 & 0.03 & 70.54 \\
\hline Capsella bursa-pastoris (L.) Med. & 6.59 & 1.36 & $\cdot 12.30$ & 7.46 & 1.30 & 0.22 & 0.02 & 0.01 & 0.02 & 0.01 & 29.28 \\
\hline Centaurea cyanus $\mathrm{L}$. & 86.15 & 24.16 & 132.24 & 65.54 & 12.44 & 2.49 & 1.28 & 0.12 & 0.45 & 0.30 & 325.08 \\
\hline Chenopodium album $\mathrm{L}$. & 54.36 & 10.57 & 73.31 & 40.85 & 8.41 & 3.28 & 0.70 & 0.02 & 0.37 & 0.04 & 191.91 \\
\hline Cirsium arvense (L.) Scop. & 74.17 & 12.02 & 100.23 & 132.01 & 21.94 & 4.94 & 0.92 & 0.07 & 0.19 & 0.16 & 346.65 \\
\hline Convolvulus arvensis $\mathrm{L}$. & 169.90 & 19.00 & 171.75 & 112.10 & 11.41 & 1.28 & 0.38 & 0.03 & 0.08 & 0.05 & 485.98 \\
\hline Galeopsis tetrahit $\mathbf{L}$. & 22.31 & 6.50 & 29.35 & 20.55 & 2.84 & 1.06 & 0.66 & 0.01 & 0.09 & 0.06 & 83.43 \\
\hline Myosotis arvensis (L.) Hill. & 14.44 & 5.83 & 27.60 & 17.52 & 2.33 & 1.12 & 0.67 & 0.01 & 0.07 & 0.03 & 69.62 \\
\hline Polygonum convolvulus $\mathrm{L}$. & 30.84 & 2.71 & 12.76 & 12.16 & 1.36 & 0.48 & 0.11 & 0.01 & 0.02 & 0.01 & 60.46 \\
\hline Polygonum lapathifolium $\mathrm{L}$. & 29.72 & 7.40 & 25.02 & 12.93 & 4.70 & 2.10 & 0.98 & 0.01 & 0.14 & 0.06 & 83.06 \\
\hline Sonchus arvensis $\mathrm{L}$. & 20.92 & 4.83 & 30.32 & 20.44 & 5.07 & 1.55 & 0.34 & 0.01 & 0.08 & 0.08 & 83.64 \\
\hline Stellaria media Vill. & 31.14 & 5.38 & 97.70 & 18.35 & 3.48 & 4.41 & 1.91 & 0.03 & 0.25 & 0.05 & 162.70 \\
\hline Veronica arvensis $\mathrm{L}$. & 4.86 & 1.17 & 7.17 & 4.77 & 1.20 & 0.24 & 0.30 & 0.01 & 0.02 & 0.01 & 19.75 \\
\hline Vicia hirsuta (L.) S.F. Gray & 72.62 & 5.82 & 33.60 & 24.17 & 4.12 & 0.84 & 0.64 & 0.03 & 0.08 & 0.10 & 142.02 \\
\hline $\begin{array}{l}\text { Viola arvensis Murr. } \\
\text { Tripleurospermum inodorum (L.) }\end{array}$ & 10.33 & 3.91 & 16.58 & 6.64 & 1.62 & 0.79 & 0.43 & 0.01 & 0.04 & 0.04 & 40.39 \\
\hline Schultz-Bip. & 50.00 & 8.06 & 86.40 & 28.78 & 5.10 & 0.47 & 1.38 & 0.04 & 0.16 & 0.11 & 180.50 \\
\hline $\mathrm{LSD}_{0.05}$ & 137.30 & - & - & 121.84 & 17.80 & 5.17 & - & 0.07 & - & - & \\
\hline
\end{tabular}




\section{Table 3}

Contents of some mineral components in spring barley (grain +straw + roots) and its weeds (the average of 1974-1976)

\begin{tabular}{|c|c|c|c|c|c|c|c|c|c|c|}
\hline \multirow{2}{*}{ Species } & \multicolumn{6}{|c|}{ Percent of dry matter } & \multicolumn{4}{|c|}{$\mathrm{mg} / \mathrm{kg}$} \\
\hline & $\mathrm{N}$ & $\mathrm{P}$ & $\mathbf{K}$ & $\mathrm{Ca}$ & $\mathrm{Mg}$ & $\mathrm{Na}$ & $\mathrm{Fe}$ & $\mathrm{Cu}$ & $\mathrm{Mn}$ & $\mathrm{Zn}$ \\
\hline Hordeum vulgare $\mathrm{L}$. & 1.50 & 0.21 & 1.36 & 0.95 & 0.13 & 0.08 & 480.0 & 9.7 & 28.6 & 41.3 \\
\hline Convolvulus arvensis $\mathrm{L}$. & 2.79 & 0.28 & 2.79 & 1.50 & 0.44 & 0.07 & 415.6 & 9.4 & 48.3 & 29.5 \\
\hline Centaurea cyanus $\mathrm{L}$. & 1.32 & 0.39 & 1.92 & 1.00 & 0.12 & 0.06 & 201.5 & 8.8 & 53.0 & 70.9 \\
\hline Chenopodium album $\mathrm{L}$. & 2.84 & 0.33 & 4.51 & 2.42 & 0.53 & 0.08 & 374.3 & 13.9 & 100.6 & 72.9 \\
\hline Galeopsis tetrahit $\mathrm{L}$. & 2.53 & 0.38 & 2.64 & 1.55 & 0.18 & 0.06 & 258.3 & 11.7 & 198.3 & 232.3 \\
\hline Polygonum convolvulus $\mathrm{L}$. & 2.91 & 0.38 & 2.93 & 3.13 & 0.98 & 0.09 & 332.2 & 10.8 & 63.0 & 74.3 \\
\hline Polygonum lapathifolium $\mathrm{L}$. & 2.30 & 0.41 & 3.32 & 2.27 & 0.65 & 0.04 & 144.0 & 11.3 & 52.0 & 31.6 \\
\hline Sonchus asper (L.) Hill. & 2.22 & 0.39 & 3.89 & 2.22 & 0.43 & 0.58 & 206.3 & 12.3 & 75.3 & 64.9 \\
\hline Sonchus arvensis $\mathbf{L}$. & 2.32 & 0.36 & 4.31 & 1.84 & 0.39 & 0.40 & 176.3 & 10.0 & 61.0 & 38.0 \\
\hline Stellaria media Vill. & 2.50 & 0.73 & 3.75 & 2.19 & 0.69 & 0.22 & 294.0 & 7.8 & 101.3 & 82.6 \\
\hline Vicia hirsuta (L.) S.F. Gray & 3.13 & 0.24 & 1.10 & 1.22 & 0.14 & 0.04 & 205.0 & 9.0 & 31.6 & 59.5 \\
\hline Vicia angustifolia $\mathrm{L}$. & 3.62 & 0.36 & 1.25 & 1.10 & 0.13 & 0.12 & 279.5 & 6.5 & 33.5 & 41.2 \\
\hline Tripleurospermum inodorum (L.) Schultz-Bip. & 1.88 & 0.29 & 2.24 & 1.11 & 0.18 & 0.06 & 174.3 & 5.6 & 26.3 & 46.9 \\
\hline $\mathrm{LSD}_{0.05}$ & - & 0.17 & 1.81 & 0.74 & 0.48 & 0.20 & - & - & 121.2 & - \\
\hline
\end{tabular}


particular elements (with the exception of $\mathrm{Na}$ ) as barley. Generally, the majority of differences among the plants could be placed within the margin of error, excluding $\mathrm{Na}, \mathrm{Fe}$ and $\mathrm{Mn}$.

The percentage of mineral elements in roots and leaves of sugar beets was high, and only a few weed species showed a greater concentration of some elements. For instance Cirsium arvense possessed higher contents of $\mathrm{N}, \mathrm{K}, \mathrm{Ca}$ and $\mathrm{Fe}$, whereas Sonchus asper - of $\mathrm{P}, \mathrm{K}, \mathrm{Ca}, \mathrm{Mn}, \mathrm{Zn}, \mathrm{Fe}$ and $\mathrm{Cu}$ (Table 5).

Considering the great mass of an individual sugar beet plant and the high concentration of mineral elements, sugar beet was unequalled by any weed in the quantity of macro- and microelement uptake.

Comparatively, the most dangerous competitors for sugar beet in taking mineral components from the soil were, by and large, Cirsium arvense, Chenopodium album, Sonchus asper, Sonchus arvensis and Echinochloa crus-galli (Table 6).

The species infesting winter rape were characterized by a greater percentage of the majority of the investigated mineral elements than rape itself (seads + straw + + roots). Only the dry matter of Agropyron repens, Centaurea cyanus and Tripleurospermum inodorum turned out to be poorer in nitrogen, calcium, magnesium and sodium (Table 7).

A different situation held for the quantity of mineral components in one plant, this being the result of component concentration and dry matter yields. Rape took up the greatest quantities of all of the investigated elements because of its great above- and underground mass. Among the weeds, the most "greedy" were, once more, Cirsium arvense and Chenopodium album, followed by Sonchus asper, Tripleurospermum inodorum, Stellaria media, Centaurea cyanus, Galeopsis tetrahit (Table 8).

\section{DISCUSSION}

The content of mineral components in plants is differentiated. It varies with soils, fertilization schemes, climatic conditions (Chojnacki and Boguszewski, 1971; Czuba and Gaszek, 1971; Mikłosz-Wiśniewska and Olkowski, 1981; Oświt and Sapek, 1982). Consequently, the data concerning the chemical composition of weeds presented in this paper should be considered as representative for loess soil conditions in the climate of the central Lublin Region. Additionally, they represent an unquestionable comparative value for the plant-weed relation.

Czuba and Wróbel's (1983) research showed the losses caused by defective utilization by plant cultures of soil nutrients from mineral fertilizers. The quantities of nutrients depleted by weeds vary, according to the authors, from 8 to $41 \%$ of the total uptake in a maize field, and from 20 to $48 \%$ on winter rape and sugar beet plantation. In the case of potato cultures the loss reaches $69,3 \%$ of potassium and $86,8 \%$ of magnesium. The discussed study does not offer any opportunity to evaluate the given figures since no indication as to the number or mass of weeds per unit area is included. Nevertheless, the approximation concerning the potassium and magnesium uptake by weeds infesting potato seems to be correct since 
Table 4

Uptake of mineral components by spring barley and its weeds, in mg per plant (the average of 1974-1976)

\begin{tabular}{|c|c|c|c|c|c|c|c|c|c|c|c|}
\hline Species & $\mathrm{N}$ & $\mathbf{P}$ & $\mathrm{K}$ & $\mathrm{Ca}$ & $\mathrm{Mg}$ & $\mathrm{Na}$ & $\mathrm{Fe}$ & $\mathrm{Cu}$ & $\mathrm{Mn}$ & $\mathrm{Zn}$ & Sum \\
\hline Hordeum vulgare $\mathrm{L}$. & 131.29 & 18.01 & 128.17 & 95.18 & 12.77 & 6.29 & 2.50 & 0.04 & 0.17 & 0.14 & 394.49 \\
\hline Convolvulus arvensis $\mathrm{L}$. & 77.89 & 7.64 & 84.96 & 51.41 & 10.63 & 1.89 & 1.11 & 0.04 & 0.18 & 0.09 & 235.81 \\
\hline Centaurea cyanus $\mathrm{L}$. & 38.25 & 11.23 & 51.14 & 18.06 & 2.54 & 1.40 & 1.04 & 0.03 & 0.16 & 0.24 & 124.08 \\
\hline Chenopodium album $\mathbf{L}$. & 62.20 & 6.80 & 91.93 & 52.15 & 11.54 & 2.83 & 0.88 & 0.03 & 0.20 & 0.04 & 228.60 \\
\hline Galeopsis tetrahit $\mathrm{L}$. & 66.44 & 11.24 & 78.74 & 47.02 & 5.72 & 2.03 & 1.15 & 0.03 & 0.39 & 0.15 & 212.91 \\
\hline Polygonum convolvulus $\mathrm{L}$. & 38.58 & 5.25 & 35.97 & 40.08 & 12.25 & 1.20 & 0.49 & 0.02 & 0.11 & 0.07 & 134.02 \\
\hline Polygonum lapathifolium $\mathrm{L}$. & 31.75 & 5.64 & 44.10 & 32.25 & 8.79 & 0.66 & 0.22 & 0.01 & 0.06 & 0.04 & 123.51 \\
\hline Sonchus asper (L.) Hill. & 48.23 & 8.88 & 84.78 & 48.65 & 9.33 & 12.41 & 0.90 & 0.03 & 0.11 & 0.13 & 213.45 \\
\hline Sonchus arvensis L. & 87.88 & 9.76 & 103.88 & 47.54 & 19.51 & 10.71 & 0.68 & 0.03 & 0.29 & 0.14 & 280.42 \\
\hline Stellaria media Vill. & 38.99 & 10.02 & 56.53 & 28.09 & 9.34 & 3.46 & 0.42 & 0.02 & 0.05 & 0.05 & 146.97 \\
\hline Vicia hirsuta (L.) S.F. Gray & 116.46 & 9.33 & 35.07 & 37.64 & 5.83 & 1.39 & 0.73 & 0.03 & 0.21 & 0.12 & 204.81 \\
\hline Vicia angustifolia $\mathrm{L}$. & 120.97 & 12.50 & 50.59 & 33.80 & 4.52 & 4.46 & 1.17 & 0.02 & 0.13 & 0.21 & 228.30 \\
\hline \multicolumn{12}{|c|}{ Tripleurospermum inodorum (L.) } \\
\hline Schultz-Bip. & 55.72 & 8.11 & 68.38 & 34.89 & 5.43 & 1.40 & 0.73 & 0.02 & 0.05 & 0.09 & 174.80 \\
\hline $\mathrm{LSD}_{0.05}$ & - & - & - & - & - & 8.13 & 1.45 & - & 0.26 & - & \\
\hline
\end{tabular}


Table 5

Contents of some mineral components in the sugar beet (roots and leaves) and its weeds (the average of 1974-1976)

\begin{tabular}{|c|c|c|c|c|c|c|c|c|c|c|}
\hline \multirow{2}{*}{ Species } & \multicolumn{6}{|c|}{ Percent of dry matter } & \multicolumn{4}{|c|}{$\mathrm{mg} / \mathrm{kg}$} \\
\hline & $\mathrm{N}$ & $\mathbf{P}$ & $\mathrm{K}$ & $\mathrm{Ca}$ & $\mathrm{Mg}$ & $\mathrm{Na}$ & $\mathbf{F e}$ & $\mathrm{Cu}$ & $\mathrm{Mn}$ & $\mathrm{Zn}$ \\
\hline Beta vulgaris $\mathbf{L}$. var. altissima Doll. & 2.38 & 0.28 & 3.50 & 1.10 & 0.47 & 1.39 & 96.0 & 6.1 & 172.0 & 70.7 \\
\hline Agropyron repens (L.) P.B. & 2.16 & 0.21 & 2.33 & 0.92 & 0.14 & 0.04 & 277.0 & 6.8 & 107.0 & 28.6 \\
\hline Capsella bursa-pastoris (L.) Med. & 2.28 & 0.39 & 2.69 & 1.86 & 0.21 & 0.06 & 337.6 & 7.2 & 85.0 & 30.2 \\
\hline Chenopodium album $\mathbf{L}$. & 2.80 & 0.40 & 2.51 & 2.18 & 0.41 & 0.07 & 276.2 & 6.4 & 156.0 & 50.8 \\
\hline Cirsium arvense (L.) Scop. & 2.58 & 0.23 & 5.48 & 3.34 & 0.25 & 0.07 & 206.3 & 5.6 & 153.0 & 38.8 \\
\hline Convolvulus arvensis $\mathbf{L}$. & 3.15 & 0.20 & 2.73 & 0.86 & 0.19 & 0.04 & 229.0 & 68.9 & 91.0 & 73.3 \\
\hline Echinochloa crus-galli (L.) P.B. & 2.07 & 0.21 & 2.58 & 0.33 & 0.47 & 0.04 & 149.0 & 13.5 & 197.0 & 61.3 \\
\hline Equisetum arvense $\mathbf{L}$. & 1.88 & 0.28 & 3.18 & 2.25 & 0.39 & 0.09 & 496.6 & 10.6 & 77.0 & 62.2 \\
\hline Galeopsis tetrahit $\mathbf{L}$. & 2.68 & 0.26 & 2.11 & 1.32 & 0.22 & 0.04 & 400.0 & 10.8 & 457.2 & 77.5 \\
\hline Myosotis arvensis (L.) Hill. & 2.36 & 0.42 & 2.57 & 1.43 & 0.24 & 0.08 & 823.0 & 12.4 & 119.0 & 47.3 \\
\hline Polygonum convovulus $\mathrm{L}$. & 2.63 & 0.30 & 1.00 & 1.17 & 0.32 & 0.05 & 298.6 & 62.1 & 71.0 & 34.8 \\
\hline Polygonum lapathifolium $\mathrm{L}$. & 2.14 & 0.24 & 1.39 & 1.28 & 0.33 & 0.05 & 287.6 & 8.5 & 271.1 & 60.7 \\
\hline Sonchus asper (L.) Hill. & 1.80 & 0.57 & 4.22 & 2.62 & 0.45 & 0.34 & 664.0 & 14.2 & 220.0 & 84.7 \\
\hline Sonchus arvensis $\mathrm{L}$. & 1.73 & 0.25 & 3.57 & 0.99 & 0.59 & 0.26 & 296.6 & 5.7 & 70.3 & 28.2 \\
\hline Stellaria media Vill. & 2.26 & 0.86 & 2.95 & 1.90 & 0.29 & 0.19 & 550.5 & 7.3 & 217.0 & 16.6 \\
\hline Viola arvensis Murr. & 2.48 & 0.40 & 2.87 & 0.93 & 0.30 & 0.08 & 466.0 & 5.1 & 188.3 & 106.0 \\
\hline Tripleurospermum inodorum (L.) Schultz-Bip. & 1.71 & 0.26 & 2.64 & 1.06 & 0.20 & 0.05 & 154.3 & 8.1 & 129.0 & 37.8 \\
\hline $\mathrm{LSD}_{0.05}$ & 1.07 & 0.29 & 3.46 & 2.16 & 0.32 & 0.95 & 501.0 & - & - & 70.9 \\
\hline
\end{tabular}


Table 6

Uptake of mineral components by the sugar beet and its weeds, in mg per plant (the average of 1974-1976)

\begin{tabular}{|c|c|c|c|c|c|c|c|c|c|c|c|}
\hline Species & $\mathrm{N}$ & $\mathbf{P}$ & $\mathrm{K}$ & $\mathrm{Ca}$ & $\mathrm{Mg}$ & $\mathrm{Na}$ & $\mathrm{Fe}$ & $\mathrm{Cu}$ & Mn & $\mathrm{Zn}$ & Sum \\
\hline Beta vulgaris L. var. altissima Doll. & 2731.2 & 171.1 & 4118.6 & 405.3 & 275.3 & 363.1 & 22.9 & 0.69 & 49.8 & 8.37 & 7946.36 \\
\hline Agropyron repens (L.) P.B. & 33.9 & 3.6 & 35.9 & 21.0 & 2.8 & 1.1 & 0.3 & 0.35 & 5.5 & 2.05 & 106.50 \\
\hline Capsella bursa-pastoris (L.) Med. & 32.3 & 5.6 & 37.8 & 26.9 & 3.1 & 1.0 & 0.5 & 0.02 & 0.1 & 0.07 & 107.39 \\
\hline Chenopodium album L. & 90.9 & 19.1 & 127.1 & 97.4 & 20.2 & 3.3 & 1.5 & 0.04 & 0.8 & 0.36 & 360.70 \\
\hline Cirsium arvense (L.) Scop. & 183.2 & 16.4 & 313.2 & 190.9 & 18.0 & 4.7 & 1.8 & 0.05 & 1.3 & 0.31 & 729.86 \\
\hline Convolvulus arvensis $\mathrm{L}$. & 67.8 & 4.3 & 66.2 & 18.5 & 4.1 & 1.2 & 0.9 & 0.36 & 0.2 & 0.15 & 163.31 \\
\hline Echinochloa crus-galli (L.) P.B. & 90.0 & 9.0 & 134.0 & 12.2 & 20.3 & 0.9 & 0.7 & 0.05 & 0.9 & 0.27 & 268.32 \\
\hline Equisetum arvense $\mathrm{L}$. & 28.1 & 4.5 & 48.1 & 37.7 & 5.8 & 1.6 & 1.0 & 0.03 & 0.2 & 0.02 & 127.05 \\
\hline Galeopsis tetrahit $\mathrm{L}$. & 86.1 & 8.5 & 67.5 & 44.5 & 6.5 & 1.8 & 1.1 & 0.03 & 1.1 & 0.31 & 217.44 \\
\hline Myosotis arvensis (L.) Hill. & 24.2 & 3.7 & 22.5 & 15.4 & 2.4 & 1.0 & 0.7 & 0.03 & 0.1 & 0.03 & 69.66 \\
\hline Poygonum convolvulus $\mathrm{L}$. & 70.9 & 8.5 & 26.8 & 29.3 & 9.5 & 1.5 & 1.0 & 0.21 & 0.2 & 0.14 & 148.05 \\
\hline Polygonum lapathifolium $\mathrm{L}$. & 83.5 & 10.8 & 64.6 & 48.4 & 14.5 & 2.0 & 1.6 & 0.09 & 1.5 & 0.42 & 227.23 \\
\hline Sonchus asper (L.) Hill. & 59.1 & 18.3 & 133.6 & 81.1 & 13.9 & 9.1 & 2.3 & 0.05 & 0.7 & 0.33 & 318.48 \\
\hline Sonchus arvensis $\mathbf{L}$. & 60.2 & 12.2 & 147.4 & 64.4 & 14.6 & 5.3 & 1.5 & 0.02 & 0.4 & 0.25 & 306.27 \\
\hline Stellaria media Vill. & 51.2 & 21.4 & 80.1 & 47.4 & 7.1 & 4.6 & 1.4 & 0.04 & 0.4 & 0.18 & 163.82 \\
\hline Viola arvensis Murr. & 22.3 & 4.5 & 30.7 & 11.9 & 2.6 & 1.2 & 0.4 & 0.03 & 0.3 & 0.04 & 73.97 \\
\hline \multicolumn{12}{|l|}{ Tripleurospermum inodorum (L). } \\
\hline Schultz-Bip. & 57.2 & 10.0 & 97.6 & 40.3 & 7.7 & 2.3 & 0.6 & 0.05 & 0.4 & 0.20 & 216.35 \\
\hline $\mathrm{LSD}_{0.05}$ & 2280.6 & 132.6 & 3599.2 & 177.0 & 221.3 & 26.0 & 14.9 & - & 32.0 & 5.81 & \\
\hline
\end{tabular}


Table 7

Contents of some mineral components in winter rape (seeds + straw + roots) and its weeds (the average of 1974-1976)

\begin{tabular}{|c|c|c|c|c|c|c|c|c|c|c|}
\hline \multirow{2}{*}{ Species } & \multicolumn{6}{|c|}{ Percent of dry matter } & \multicolumn{4}{|c|}{$\mathrm{mg} / \mathrm{kg}$} \\
\hline & $\mathrm{N}$ & $\mathbf{P}$ & $\mathbf{K}$ & $\mathrm{Ca}$ & $\mathrm{Mg}$ & $\mathrm{Na}$ & $\mathrm{Fe}$ & $\mathrm{Cu}$ & $\mathrm{Mn}$ & $\mathrm{Zn}$ \\
\hline $\begin{array}{l}\text { Brassica napus L. var. napus } f . \text { biennis } \\
\text { (Schubl. et Mart.) Thell. }\end{array}$ & 1.81 & 0.29 & 1.18 & 1.37 & 0.16 & 0.14 & 157.0 & 5.0 & 64.0 & 50.6 \\
\hline Agropyron repens (L.) P.B. & 1.52 & 0.29 & 2.08 & 0.49 & 0.12 & 0.03 & 544.0 & 5.6 & 68.3 & 56.6 \\
\hline Capsella bursa-pastoris (L.) Med. & 1.90 & 0.31 & 1.88 & 1.58 & 0.19 & 0.05 & 520.6 & 5.0 & 104.3 & 57.5 \\
\hline Centaurea cyanus $\mathrm{L}$. & 1.67 & 0.36 & 1.46 & 0.65 & 0.18 & 0.04 & 146.6 & 9.4 & 61.3 & 35.8 \\
\hline Chenopodium album $\mathrm{L}$. & 3.49 & 0.65 & 3.98 & 3.89 & 0.81 & 0.11 & 352.5 & 9.7 & 89.0 & 36.0 \\
\hline Cirsium arvense (L.) Scop. & 2.62 & 0.64 & 3.10 & 4.04 & 0.44 & 0.13 & 426.0 & 13.6 & 91.3 & 48.9 \\
\hline Convolvulus arvensis L. & 2.60 & 0.28 & 1.92 & 0.93 & 0.27 & 0.03 & 589.0 & 7.7 & 37.3 & 41.3 \\
\hline Equisetum arvense L. & 2.23 & 0.33 & 2.09 & 1.71 & 0.35 & 0.02 & 746.0 & 10.3 & 45.0 & 44.3 \\
\hline Galeopsis tetrahit $\mathbf{L}$. & 2.91 & 0.42 & 2.58 & 1.43 & 0.21 & 0.05 & 622.3 & 8.6 & 247.6 & 77.6 \\
\hline Myosotis arvensis (L.) Hill. & 1.81 & 0.54 & 3.32 & 1.51 & 0.20 & 0.08 & 475.6 & 9.4 & 115.3 & 44.3 \\
\hline Polygonum convolvulus $\mathrm{L}$. & 2.94 & 0.47 & 2.53 & 1.39 & 0.38 & 0.07 & 647.6 & 9.4 & 138.0 & 73.6 \\
\hline Polygonum lapathifolium L. & 2.07 & 0.41 & 2.06 & 1.25 & 0.35 & 0.04 & 692.0 & 13.5 & 150.0 & 96.0 \\
\hline Sonchus asper (L.) Hill. & 2.13 & 0.42 & 4.17 & 1.73 & 0.38 & 0.11 & 404.0 & 8.1 & 222.0 & 53.0 \\
\hline Sonchus arvensis $\mathrm{L}$. & 2.45 & 0.40 & 5.27 & 1.79 & 0.36 & 0.10 & 869.0 & 8.5 & 274.5 & 71.0 \\
\hline Stellaria media Vill. & 2.85 & 0.67 & 4.10 & 1.38 & 0.26 & 0.23 & 392.0 & 7.9 & 235.6 & 70.6 \\
\hline Vicia hirsuta (L.) S.F. Gray & 3.13 & 0.35 & 2.02 & 0.96 & 0.21 & 0.04 & 227.6 & 9.9 & 46.3 & 46.0 \\
\hline Viola arvensis Murr. & 1.91 & 0.30 & 2.75 & 0.95 & 0.21 & 0.04 & 326.3 & 8.5 & 143.0 & 103.6 \\
\hline Tripleurospermum inodorum (L.) Schultz-Bip. & 1.63 & 0.42 & 2.82 & 1.06 & 0.23 & 0.08 & 243.3 & 9.8 & 89.0 & 54.8 \\
\hline $\mathrm{LSD}_{0.05}$ & 1.02 & 0.33 & 1.66 & 1.61 & 0.20 & 0.12 & 687.9 & - & 158.4 & 68.5 \\
\hline
\end{tabular}


Table 8

Uptake of mineral components by winter rape and its weeds, in mg per plant (the average of 1974-1976)

\begin{tabular}{|c|c|c|c|c|c|c|c|c|c|c|c|}
\hline Species & $\mathrm{N}$ & $\mathbf{P}$ & $\mathrm{K}$ & $\mathrm{Ca}$ & $\mathrm{Mg}$ & $\mathrm{Na}$ & $\mathrm{Fe}$ & $\mathrm{Cu}$ & $\mathrm{Mn}$ & $\mathrm{Zn}$ & Sum \\
\hline $\begin{array}{l}\text { Brassica napus L. var. napus f. } \\
\text { biennis (Schubl. et Mart.) } \\
\text { Thell. }\end{array}$ & 251.83 & 48.57 & 164.59 & 176.39 & 25.01 & 17.37 & 2.86 & 0.08 & 0.78 & 0.65 & 688.43 \\
\hline Agropyron repens (L.) P.B. & 30.62 & 6.94 & 57.00 & 16.06 & 3.29 & 1.23 & 0.51 & 0.03 & 0.15 & 0.02 & 115.95 \\
\hline Capsella bursa-pastoris (L.) Med. & 20.69 & 3.20 & 19.44 & 15.77 & 1.72 & 0.66 & 0.45 & 0.01 & 0.08 & 0.05 & 62.07 \\
\hline Centaurea cyanus $\mathrm{L}$. & 57.60 & 13.03 & 54.27 & 22.28 & 6.87 & 1.82 & 0.90 & 0.05 & 0.24 & 0.16 & 157.22 \\
\hline Chenopodium album $\mathbf{L}$. & 92.52 & 22.40 & 86.10 & 114.00 & 18.15 & 4.75 & 1.37 & 0.02 & 0.28 & 0.28 & 339.87 \\
\hline Cirsium arvense (L.) Scop. & 78.29 & 20.53 & 112.66 & 115.15 & 21.70 & 5.42 & 1.91 & 0.05 & 0.28 & 0.20 & 356.19 \\
\hline Convolvulus arvensis $\mathrm{L}$. & 30.33 & 2.82 & 20.63 & 10.70 & 3.76 & 0.18 & 1.01 & 0.02 & 0.08 & 0.07 & 69.60 \\
\hline Equisetum arvense $\mathrm{L}$. & 36.60 & 5.40 & $34: 20$ & 28.00 & 5.82 & 0.49 & 1.80 & 0.02 & 1.10 & 0.09 & 113.53 \\
\hline Galeopsis tetrahit L. & 56.52 & 7.79 & 45.82 & 33.36 & 5.71 & 1.37 & 0.76 & 0.01 & 0.32 & 0.09 & 151.75 \\
\hline Myosotis arvensis (L.) Hill. & 15.53 & 5.17 & 52.33 & 15.38 & 1.93 & 1.07 & 0.39 & 0.01 & 0.10 & 0.03 & 91.94 \\
\hline Polygonum convolvulus L. & 24.71 & 2.56 & 18.45 & 15.30 & 3.01 & 0.49 & 0.56 & 0.02 & 0.12 & 0.04 & 65.26 \\
\hline Polygonum lapathifolium $\mathrm{L}$. & 14.28 & 2.57 & 14.89 & 8.98 & 3.69 & 0.19 & 0.56 & 0.02 & 0.20 & 0.13 & 45.51 \\
\hline Sonchus asper (L.) Hill. & 41.38 & 7.36 & 95.18 & 40.72 & 5.71 & 2.48 & 1.64 & 0.03 & 0.47 & 0.19 & 195.56 \\
\hline Sonchus arvensis L. & 32.00 & 4.21 & 66.46 & 18.23 & 5.19 & 1.60 & 1.62 & 0.01 & 0.37 & 0.16 & 129.85 \\
\hline Stellaria media Vill. & 55.33 & 13.91 & 77.57 & 27.32 & 4.65 & 4.53 & 0.76 & 0.02 & 0.49 & 0.15 & 184.73 \\
\hline Vicia hirsuta (L.) S. F. Gray & 48.75 & 5.04 & 34.63 . & 17.42 & 5.12 & 0.68 & 0.50 & 0.01 & 0.06 & 0.07 & 112.28 \\
\hline Viola arvensis Murr. & 15.69 & 2.31 & 17.19 & 9.50 & 1.73 & 0.40 & 0.14 & 0.01 & 0.37 & 0.10 & 47.44 \\
\hline $\begin{array}{l}\text { Tripleurospermum inodorum (L). } \\
\text { Schultz-Bip. }\end{array}$ & 46.22 & 13.34 & 82.62 & 41.19 & 6.87 & 4.04 & 0.99 & 0.04 & 0.26 & 0.11 & 195.72 \\
\hline $\mathrm{LSD}_{0.05}$ & 86.29 & 25.80 & 115.13 & 71.10 & 14.71 & 5.81 & 1.70 & 0.07 & 0.68 & 0.03 & \\
\hline
\end{tabular}


it refers to Chenopodium album - the species characterized also in Czesławice by a high uptake and percentage of mineral elements in dry matter. Similarly, in Świętochowski and Sonta's (1962) experiments, Chenopodium album, of all of the investigated species, developed the most abundant mass and gathered the greatest quantity of nutrients even in conditions of unfavourable humidity, an analogical situation appeared in the case of Sonchus arvensis, which is also mentioned in the quoted work.

Li-Sun-Żun (1962) found in Echinochloa crus-galli - as much as 5,3\% nitrogen and $6,1 \%$ potassium. These results, however, do not correspond with ours, doubling the figures we have arrived at. Nevertheless, it does not undermine the ascertainment that weeds contain much greater quantities of macro- and microelements than cultivated plants. Thus Shukla and Vimal (1969) are undoubtedly correct when they advise to compost weeds since, utilized as fertilizer, they increase the content of available NPK forms in the soil.

\section{CONCLUSIONS}

1. The mass unit of the majority of field weeds contains many more mineral components than that of cultivated plants. An especially high percentage of macroand microelements is found in: Chenopodium album, Sonchus arvensis, Sonchus asper, Cirsium arvensis, Polygonum convolvulus, Polygonum lapathifolium, Convolvulus arvensis, Stellaria media, Echinochloa crus-galli and in Galeopsis tetrahit.

2. The most dangerous competitors for the uptake of nutrients in loess soil culture are: Chenopodium album, Cirsium arvense, Convolvulus arvensis, Sonchus arvensis, Stellaria media and Polygonum convolvulus. Consequently, one must pay particular attention to just these species when selecting weeding methods.

\section{REFERENCES}

Chojnacki A., Boguszewski W., 1971. Zawartość azotu, fosforu i potasu w głównych roślinach uprawnych w Polsce. Pam. Pul. 50: 5-27.

Czuba R., Gaszek K., 1971. Wpływ mikronawozów na zawartość przyswajalnych form mikroelementów $w$ profilu glebowym oraz na skład chemiczny roślin. Rocz. Nauk Roln. A-97-4: $75-89$.

Czuba R., Wróbel S., 1983. Konkurencyjność chwastów w pobieraniu składników pokarmowych przez rośliny uprawne. Nowe Roln. 2: 8-11.

Li-Sun-Żun, 1962. Badania ekologiczne nad chwastnicą jednostronną - Echinochloa crus-galli (L.) var. longisetum DÖLL. Rocz. Nauk Roln. A-86-1: 1-29.

Mikłosz-Wiśniewska St., Olkowski M., 1981. Kształtowanie się zawartości niektórych składników mineralnych $w$ stokłosie bezostnej i perzu właściwym pod wpływem zróżnicowanego nawożenia mineralnego. Zesz. Nauk. ART w Olsztynie, Rolnictwo 31: 133-140.

Mowszowicz J,, 1975. Krajowe chwasty polne i ogrodowe. Wyd. II. PWRiL, Warszawa.

Oświt J., Sa pek S., 1982. Ocena zawartości składników mineralnych w roślinach łąk naturalnych i zdolność gatunków do wykorzystania zasobów glebowych. Rocz. Glebozn. 33: 145-151. 
Shukla G. G., Vimal O. P., 1969. Chemical analysis of some weeds and the release of N, P and K on their addition to soil. The Indian J. of Agric. Science, 39, 2: 162-179.

Świętochowski B., Sońta W., 1962. Wpływ wilgotności gleby na wzrost i rozwój niektórych chwastów segetalnych (Doświadczenie wazonowe). Rocz. Nauk Roln. A-85-1: 1-28.

Pobieranie ważniejszych składników mineralnych przez pospolite chwasty polne gleb lessowych

\section{Streszczenie}

W latach 1974-1976 oznaczono procentową zawartość N, P, K, Ca, Mg, Na, Fe, Cu, Mn i $\mathrm{Zn}$ w częściach nadziemnych i korzeniach pszenicy ozimej, jęczmienia jarego, buraka cukrowego i rzepaku ozimego, jak również $w$ najpospolitszych gatunkach chwastów tych upraw. Materiał do analiz zbierano w fazie zawiązywania nasion przez chwasty. Azot określono metodą Kjeldahla w próbkach zmineralizowanych na mokro, zaś zawartość pozostałych składników - w próbkach spopielonych. Przy tym P oznaczono kolorymetrycznie, a inne pierwiastki na spektrometrze absorpcji atomowej. Uwzględniając średnią masę jednej rośliny, wyliczono ilość pierwiastków pobranych przez chwasty i rośliny uprawne.

Stwierdzono, że jednostka masy większości gatunków chwastów polnych zawiera więcej składników mineralnych niż jednostka masy roślin uprawnych. Szczególnie wysoką zawartością makroi mikroelementów odznaczają się: Chenopodium album, Sonchus arvensis, Sonchus asper, Cirsium arvense, Convolvulus arvensis, Polygonum convolvulus, Polygonum lapathifolium, Stellaria media, Echinochloa crus-galli oraz Galeopsis tetrahit. Najgroźniejszymi konkurentami glównych kultur gleb lessowych pod względem pobierania składników pokarmowych są: Chenopodium album, Cirsium arvense, Convolvulus arvensis, Sonchus arvensis, Stellaria media i Polygonum convolvulus. $\mathrm{Na}$ te gatunki trzeba więc w pierwszej kolejności zwrócić uwage przy doborze metody walki z chwastami. 